Tôhoku Math Journ.

23(1971), 581-588

\title{
EXT-PRODUCTS AND EDGE-MORPHISMS
}

\author{
J.GAMST AND K.HOECHSMANN
}

(Received January 12, 1970)

In a previous paper, we constructed products for certain Ext-functors, in particular for $\operatorname{Ext}^{*}\left(A_{X},-\right)=H^{*}(X,-)$ where $A_{x}$ denotes the structure sheaf of an object $X$ in a ringed site. If $U$ is a covering of $X$, it must be asked whether our pairings have anything to do with the usual cup-product in the $\dot{\mathrm{C} E C H}$ cohomology $H^{*}(U,-)$. The answer is that the two are related by the edge-morphism $H^{*}(U,-)$ $\rightarrow H^{*}(X,-)$ of the CARTAN-LERAY spectral sequence.

To see this, we proceed in two stages. In section 2 , we show that our products (whose construction is sketched at the beginning of that section) are compatible with edge-morphisms arising from an adjoint situation. The main work is in section 1, which gives a very explicit description of such edge-morphisms.

In section 3, we look at $H^{q}(U,-)$ as an Ext-functor on the category of presheaves and show that the pairing defined by our constructions coincides with the simplicial cup-product.

We shall adhere to the notational conventions of [2]. For the sake of completeness, let us list the most important ones again. Consider an abelian category $\mathcal{A}$. By $K(\mathcal{A})$ we denote the category of (co-chain) complexes in $\mathcal{A}$ and homotopy classes of maps, specifying the full subcategory of complexes bounded below (resp. above) by a superscript + (resp. - ). $D(\mathcal{A})$ stands for the derived category of $A$ : it is obtained from $K(\mathcal{A})$ by formally inverting those $K(\mathscr{A})$ -arrows which induce isomorphisms in the cohomology. As in [2], the latter will be called quisos and represented by double arrows. Thus, morphisms in $D(\mathcal{A})$ are given as left or right fractions with quisos as "denominators." If $u$ : $\mathscr{A} \rightarrow \mathscr{B}$ is an additive functor to another abelian category, a left $u$-acyclic class $\mathscr{P} \subset \mathcal{A}$ is a full subcategory such that $u$ respects quisos between objects of $K^{-}(\mathscr{Q})$ and such that every object $M^{*}$ of $K^{-}(\mathcal{A})$ admits a quiso $P^{*} \Longrightarrow M^{*}$ with $P^{*}$ in $K^{-}(\mathscr{Q})$. In the usual way, $\mathscr{P}$ permits the construction of a left derived functor $\mathcal{L} u: D^{-}(\mathcal{A})$ $\rightarrow D(\mathscr{B})$. The obvious dual notion of right u-acyclic class is analogously related to the existence of a right derived functor $\mathcal{R} u: D^{+}(\mathscr{A}) \rightarrow D(\mathscr{B})$.

1. Some edge morphisms. Throughout this section, we fix our attention on a pair $u, v$ of additive functors between abelian categories 


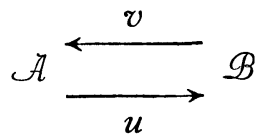

supposing $u$ to be left adjoint to $v$ and assuming the existence of a left $u$-acyclic class $\mathscr{P} \subset \mathcal{A}$ and of a right $v$-acyclic class $\mathscr{g} \subset \mathscr{B}$.

We begin by extending the given adjointness

$$
\operatorname{Hom}_{\mathcal{A}}(X, v Y) \underset{\sim}{\rightarrow} \operatorname{Hom}_{\mathcal{B}}(u X, Y)
$$

to the derived categories. Given a morphism

$$
P^{*} \Leftarrow Q^{*} \rightarrow v N^{*}
$$

in $D(\mathscr{A})$ with $P^{*}, Q^{*} \in D^{-}(\mathscr{Q})$ and $N^{*} \in D^{+}(\mathscr{B})$, we apply $u$ and the canonical adjunction map $w v \rightarrow I d_{\mathcal{B}}$ to obtain the morphism

$$
u P^{*} \Leftarrow u Q^{*} \rightarrow u v N^{*} \rightarrow N^{*} .
$$

This procedure gives us a natural map

$$
\alpha_{P^{*}, N^{*}}: \operatorname{Hom}_{D(\mathcal{A})}\left(P^{*}, v N^{*}\right) \rightarrow \operatorname{Hom}_{D(\mathcal{B})}\left(u P^{*}, N^{*}\right)
$$

for left $u$-acyclic $P^{*}$ and arbitrary $N^{*}$ (bounded to the right and to the left respectively). Dually, for $M^{*} \in D^{-}(\mathcal{A})$ and $J^{*} \in D^{+}(\mathscr{g})$ we have

$$
\beta_{M^{*}, J^{*}}: \operatorname{Hom}_{D(\mathscr{B})}\left(u M^{*}, J^{*}\right) \rightarrow \operatorname{Hom}_{D(\mathcal{H})}\left(\mathrm{M}^{*}, v J^{*}\right) .
$$

PROPOSITION 1.1. If $P^{*} \in D^{-}(\mathscr{Q})$ and $J^{*} \in D^{+}(g)$, the respective maps $\alpha$ and $\beta$ are inverses of each other. Hence $\alpha$ induces natural isomorphisms

$$
\gamma_{M^{*}, N^{*}}: \operatorname{Hom}_{D(\mathscr{B})}\left(\mathcal{L} u \mathrm{M}^{*}, N^{*}\right) \simeq \operatorname{Hom}_{D(\mathcal{G})}\left(M^{*}, \mathscr{R} v N^{*}\right)
$$

ProOF. Consider the morphism (1) with $N^{*}=J^{*}$, apply $\alpha$ to obtain (2), and represent the lattter as a left fraction $u P^{*} \rightarrow K^{*} \Longleftarrow J^{*}$ with $K^{*} \in D^{+}(\mathscr{B})$. This yields the commutative square

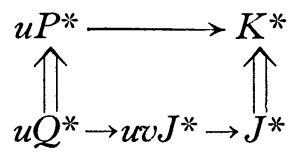


Applying $v$ and the adjunction map $I d_{\mathcal{A}} \rightarrow v u$, we get

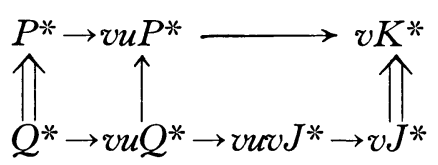

a morphism in $D(\mathcal{A})$ from $P^{*}$ to $v J^{*}$ which represents the application of $\beta \cdot \alpha$ to (1). Since the bottom line in (3) is the same as the original arrow $Q^{*} \rightarrow v J^{*}$, we indeed have $\beta \cdot \alpha=$ identity. The other composite $\alpha \cdot \beta$ is treated analogously. The second half of the proposition follows immediately from the definition of $\mathscr{R} v$ and $\mathcal{L} u$ in terms of $g$ and $\mathcal{L}($ cf. [ 3] or [2]).

The aim of this section is to identify $\alpha$ with a certain edge-morphism. For this purpose we recall (cf. [3]) that

$$
\operatorname{Ext}_{\mathcal{A}}^{P}\left(M^{*}, L^{*}\right) \cong \operatorname{Hom}_{\mathcal{A}}\left(M^{*}, L^{*}[p]\right)
$$

where $M^{*} \in D^{-}(\mathcal{A}), L^{*} \in D^{+}(\mathcal{A})$ and $[p]$ denotes a $p$-fold shift : $\left(L^{*}[p]\right)^{n}=L^{n+p}$. We now restrict ourselves to objects $P$ of $\mathscr{P}$ and $N$ of $\mathscr{B}$ and consider the commutative diagram

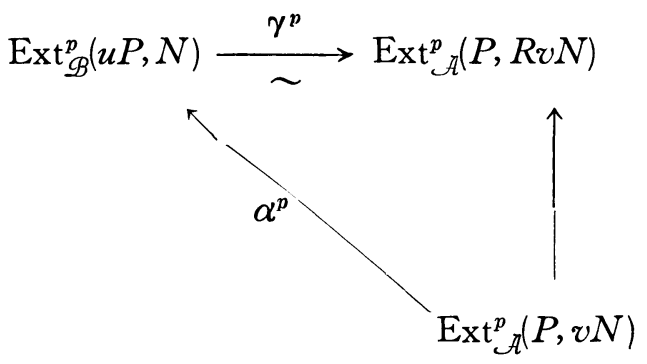

in which the vertical arrow comes from the canonical morphism $v \rightarrow \mathscr{R v}$, and $\alpha^{p}, \gamma^{p}$ correspond to the previously constructed $\alpha, \gamma$ in an obvious way $(\mathcal{L} u P \rightarrow u P$ being a quiso).

PROPOSITION 1.2. If $\mathcal{A}$ and $\mathscr{B}$ have enough injectives, there are natural convergent spectral sequences

$$
\operatorname{Ext}_{\mathcal{A}}^{p}\left(P, R^{q} v N\right) \Longrightarrow \operatorname{Ext}_{\mathscr{B}}^{p+p}(u P, N)
$$

provided that $P$ is left u-acyclic. The morphisms $\alpha^{p}$ of $(4)$ coincide with the corresponding edge-morphisms of ( 5 ). 
Proof. By Proposition 1.1, we have for injectives $I$ of $\mathscr{B}$ :

$$
\begin{aligned}
0=\operatorname{Ext}_{\mathscr{B}}^{p}(u P, I) & =\operatorname{Hom}_{D_{\mathcal{B}}}(u P, I[p]) \\
& \cong \operatorname{Hom}_{D_{\mathcal{A}}}(P, v I[p])=\operatorname{Ext}_{\mathcal{A}}^{p}(P, v I) .
\end{aligned}
$$

Hence $v I$ is acyclic for $\operatorname{Hom}_{\mathcal{A}}(P,-)$. Considering $\operatorname{Hom}_{\mathcal{B}}(u P,-)$ to be the composite $\operatorname{Hom}_{\mathcal{A}}(P,-) \circ v$, we obtain (5) as usual via a CARTAN-EILENBERG resolution.

In terms of derived functors, we have

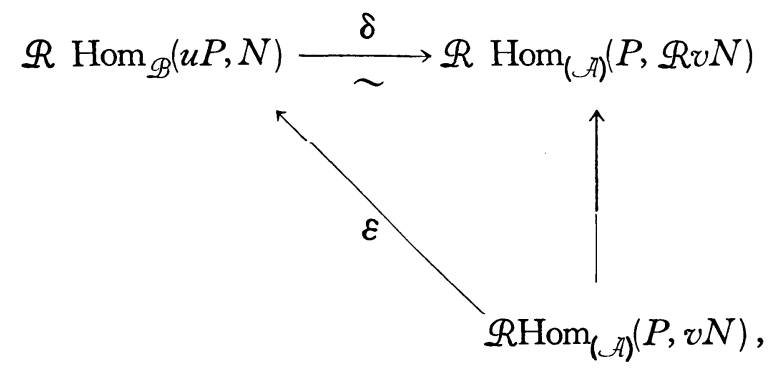

where the horizontal arrow $\delta$ represents the canonical morphism

$$
\mathscr{R}\left(\operatorname{Hom}_{\mathcal{A}}(P,-) \cdot v\right) \rightarrow \mathcal{R H o m}_{\mathcal{A}}(P,-) \cdot \mathscr{R} v \text {. }
$$

In this situation it is well-known that the maps induced in cohomology by the morphism $\varepsilon$ of $(6)$ are edge-morphisms of $(5)$. To see that these coincide with the $\alpha^{p}$ of $(4)$, it is necessary to identify $(4)$ as the diagram induced by (6) in cohomology; i. e. to show that $\delta$ induces the $\gamma^{p}$. This is easily checked by viewing $\delta$ as

$$
\delta: \operatorname{Hom}_{\mathscr{B}}^{*}\left(u P, I^{*}\right) \underset{\rightarrow}{\rightarrow} \operatorname{Hom}_{\mathscr{B}}^{*}\left(P, v I^{*}\right)
$$

where $N \Longrightarrow I^{*}$ is an injective resolution (remember that $v I^{*}$ is acyclic for $\left.\operatorname{Hom}_{\mathcal{A}}(P,-)\right)$.

2. Compatibility with Ext-products. We keep the basic situation

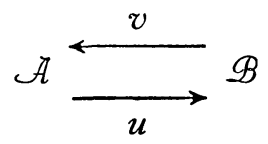

of section 1 and add the following hypotheses:

P 1: Both $\mathcal{A}$ and $\mathscr{B}$ have tensor-products in the sense of [2] (right-exact, bi-additive bifunctors with the usual symmetries). 
P 2: Both $\mathcal{A}$ and $\mathscr{B}$ have enough flats as well as enough injectives.

Under these conditions, we can tensor morphisms in $D(\mathscr{A})$ and $D(\mathscr{B})$. Given for instance

$$
P_{1}^{*} \longleftarrow Q_{1}^{*} \rightarrow L_{1}^{*} \text { and } P_{2}^{*} \Longleftarrow Q_{2}^{*} \rightarrow L_{2}^{*}
$$

with $P_{i}^{*}, Q_{i}^{*}$ flat, we can make

$$
P_{1}^{*} \otimes P_{2}^{*} \Leftarrow Q_{1}^{*} \otimes Q_{2}^{*} \rightarrow L_{1}^{*} \otimes L_{2}^{*} .
$$

In particular, we get products for $\operatorname{Ext}_{(A)}^{*}$ by its interpretation as morphisms in $D(A)$.

To apply the results of section 1 , we make the additional assumptions:

C 1: There are natural isomorphisms

$$
u\left(M_{1} \otimes M_{2}\right) \stackrel{\sim}{\rightarrow} u M_{1} \otimes u M_{2}
$$

C 2: The flats of $\mathcal{A}$ are left $u$-acyclic.

C 3: $u$ takes flats of $\mathcal{A}$ into flats of $\mathscr{B}$.

By $\mathrm{C} 1$ and adjointness, one obtains natural pairings

$$
v N_{1} \otimes v N_{2} \rightarrow v\left(N_{1} \otimes N_{2}\right)
$$

for arbitrary $N_{1}, N_{2}$ of $\mathscr{B}$. Hence for flat objects $P_{1}, P_{2}$ of $\mathcal{A}$ one has products

$$
\operatorname{Ext}_{\mathcal{A}}^{p}\left(P_{1}, v N_{1}\right) \times \operatorname{Ext}_{\mathcal{A}}^{q}\left(P_{2}, v N_{2}\right) \rightarrow \operatorname{Ext}_{\mathcal{A}}^{p+q}\left(P_{1} \otimes P_{2}, v\left(N_{1} \otimes N_{2}\right)\right)
$$

and also(on account of $\mathrm{C} 1$ and $\mathrm{C} 3)$

$$
\operatorname{Ext}_{\mathcal{B}}^{p}\left(u P_{1}, N_{1}\right) \times \operatorname{Ext}_{\mathscr{B}}^{q}\left(u P_{2}, N_{2}\right) \rightarrow \operatorname{Ext}_{\mathscr{B}}^{p+q}\left(u\left(P_{1} \otimes P_{2}\right), N_{1} \otimes N_{2}\right)
$$

Proposition 2.1. The edge-morphisms $\alpha^{p}, \alpha^{q}, \alpha^{p+q}$ of Proposition 1.2 map the product ( 7 ) onto the product ( 8 ).

The proof is a routine verification using the product-construction given at the beginning of this section and the explicit from of $\alpha$ given prior to Proposition 1.1.

3. Application to sheaves. The situation of sections 1 and 2 arises in a natural way from a morphism

$$
(u, \varphi):(\mathscr{X}, A) \rightarrow(, \mathscr{Y} B)
$$

of ringed sites by way of the associated inverse and direct image functors 


$$
u^{*}: \mathscr{A} \rightarrow \mathscr{B}, u_{*}: \mathscr{B} \rightarrow \mathcal{A}
$$

between the categories $\mathcal{A}$ and $\mathscr{B}$ of sheaves of modules. It is well-known [5], that $u^{*}$ is left adjoint to $u_{*}$ and that conditions $\mathrm{P} 1, \mathrm{P} 2, \mathrm{C} 1$ of section 2 are satisfied. Elsewhere [1] it will be shown that C 2 and $\mathrm{C} 3$ also hold.

Applying our results to this set-up, we obtain natural convergent spectral sequences

$$
\operatorname{Ext}_{\mathcal{A}}^{p}\left(P, R^{q} u_{*} F\right) \Longrightarrow \operatorname{Ext}_{\mathscr{B}}^{p+q}\left(u^{*} P, F\right)
$$

for flat $A$-modules $P$ and arbitrary $B$-modules $F$. This generalizes proposition 5.5 of exposé $\bar{Q}$ in [5]. Moreover, the edge-morphisms

$$
\operatorname{Ext}_{\mathcal{A}}^{p}\left(P, u_{*} F\right) \rightarrow \operatorname{Ext}_{\mathscr{B}}^{p}\left(u^{*} P, F\right)
$$

respect the Ext-products defined in [2]. In particular, since $u^{*} B=A$, the products induced on the sheaf-cohomology are compatible via the maps

$$
H^{p}\left(X, u_{*} F\right) \rightarrow H^{p}(Y, F)
$$

For the remainder of this section, we shall be concerned with the transition from presheaves to sheaves on a fixed ringed site $(\mathscr{X}, A)$, where $\mathscr{X}$ has pull-backs. Denoting presheaves by $\mathcal{A}$ and sheaves by $\mathscr{B}$, the canonical pair

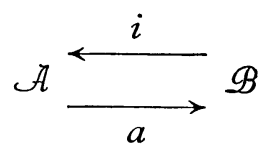

"inclusion" and "association" can be viewed as direct image and inverse image (respectively) of the morphism of sites

$$
\left(1_{X^{\prime}} \Phi\right):\left(\mathscr{X}_{\mathcal{C}}, i A\right) \rightarrow(\mathscr{X}, A),
$$

where $\mathscr{X}_{\mathcal{C}}$ denotes the category $\mathscr{X}$ equipped with the "chaotic" topology and $\Phi: A \rightarrow a i A$ an adjunction morphism.

The forgetful functor from $\mathcal{A}$ to presheaves of sets has a left adjoint $f: H \rightarrow A_{H}$ whose values are flat $i A$-modules. We are interested in $\operatorname{Ext}_{\mathcal{A}}^{p}\left(A_{R}, F\right)$ where $R$ denotes a covering crible for an object $X \in \mathscr{X}$. Remembering that, for representable $H, f(H)=A_{H}$ is a projective $i A$-module, we can compute this Ext-group via the projective resolution $R_{*}(U) \Longrightarrow A_{R}$ obtained from a generating family $U=\left\{U_{i} \rightarrow X\right\}$ for $R$ by applying $f$ to the complex 


$$
\text { .. II } U_{i} \underset{\mathbf{x}}{U_{j} \times U_{\mathbf{x}}} \stackrel{\rightarrow}{\rightarrow} \text { II } U_{i} \underset{x}{\times} U_{j} \rightarrow \text { II } U_{i} \rightarrow R .
$$

Thus, $\operatorname{Ext}_{\mathcal{A}}^{p}\left(A_{R}, F\right)=H^{p}\left(\operatorname{Hom}_{\mathcal{A}}^{*}\left(R_{*}(U), F\right)\right.$ is the "Cech-cohomology" of the covering $U$ which as usual is denoted by $H^{p}(U, F)$. Therefore (9) turns into the spectral sequence

$$
H^{p}\left(U, \mathcal{H}^{q}(F)\right) \Longrightarrow H^{p+q}(X, F)
$$

of CARTAN-LERAY.

To interpret our results concerning products, we use the canonical isomorphisms

$$
A_{B} \otimes A_{K} \underset{\rightarrow}{\sim} A_{H \times K}
$$

(cf. 2.13 in exposé IV of [5]) and the diagonal

$$
A_{H} \rightarrow A_{H \times H}
$$

to obtain pairings

$$
\begin{gathered}
H^{p}(U, i F) \otimes H^{q}(U, i G) \rightarrow H^{p+q}(U, i(F \otimes G)) \\
H^{p}(X, F) \otimes H^{q}(X, G) \rightarrow H^{p+q}(X, F \otimes G)
\end{gathered}
$$

from the Ext-products of [2]. In these terms we have:

PROPOSITION 3.1.

a) The edge-morphism $H^{p}(U, i F) \rightarrow H^{p}(X, F)$ of (10) maps the product (11) into the product (12).

b) The product (11) coincides with the usual cup-product.

PROOF. Since only (b) remains to be proved, we shall stay in $\mathcal{A}$ from now on. Whenever we can work with projective resolutions, our Ext-product clearly coincides with the "external product" of [4], Chp. VIII, §4. To see this, one has only to recall the connection between Ext and morphisms in the derived category as exhibited by means of a projective resolution. In the present case, the external product turns pairs of cocycles of $\operatorname{Hom}^{*}\left(R_{*}(U),-\right)$ into cocycles of $\mathrm{Hom}^{*}\left(R_{*}(U) \otimes R_{*}(U),-\right)$. From this same process, the usual cup-product is obtained by composition with the morphism

$$
R_{*}(U) \stackrel{\Delta}{\rightarrow} R_{*}(U \times U) \stackrel{\omega}{\rightarrow} R_{*}(U) \otimes R_{*}(U),
$$


where $\delta$ is the diagonal and $\omega$ the map produced by the ALEXANDERWHITNEY formula (cf. [4], Chp. VIII, formula (8.7)). The commutativity of

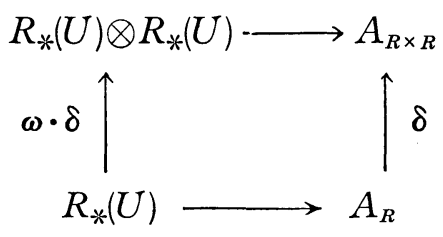

now completes the proof.

\section{REFERENCES}

[1] J. GAMST. Flat Modules on Ringed sites, to appear.

[2] J. Gamst AND K. HoechSManN. Products in Sheaf-Cohomology, Tôhoku Math. J, 22(1970).

[3] R. HARTSHORNE : Residues and Duality, Springer Lecture Notes, 20 (1966).

[4] S. MAClANE : Homology, Springer verlag, Berlin (1963).

[5] J. L. VerdiER : Cohomologie étale des schémas, SGAA 1, I. H. E. S. Paris (1963).

DEPARTMENT OF MATHEMATICS

UNIVERSITY OF BRITISH COLUMBIA

VANCOUVER, B. C., CANADA 